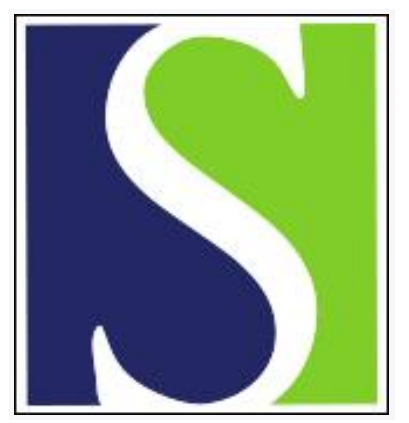

Scand J Work Environ Health 2006;32(1):75-84

https://doi.org/10.5271/sjweh.979

Issue date: 31 Feb 2006

Work ability index of young employees and their sickness absence during the following year

by Kujala V, Tammelin T, Remes J, Vammavaara E, Ek E, Laitinen J

Affiliation: Finnish Institute of Occupational Health, Aapistie 1, Fl-90220 Oulu, Finland. veikko.kujala@ttl.fi

The following articles refer to this text: 2007;33(5):351-357;

2009;35(1):37-47; 2009;35(1):56-64; 2009;35(5):325-333;

2009;35(5):321-324; 2010;36(5):404-412; 2010;36(6):515-516;

2012;38(6):560-567; 2015;41(1):36-42

Key terms: classification of occupations; control over work; longitudinal study; number of children; occupational health; physical activity; prospective population-based study; questionnaire; sex factor; sickness absence; smoking; work ability index; young employee

This article in PubMed: www.ncbi.nlm.nih.gov/pubmed/16539175

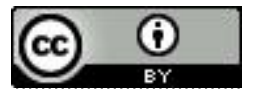




\title{
Work ability index of young employees and their sickness absence during the following year
}

\author{
by Veikko Kujala, MD, ${ }^{1,2}$ Tuija Tammelin, PhD, ${ }^{1}$ Jouko Remes, MSc, ${ }^{1}$ Elina Vammavaara, MSc, ${ }^{1}$ Ellen Ek, \\ LicA, ${ }^{1}$ Jaana Laitinen, $P h D^{1,3}$
}

Kujala V, Tammelin T, Remes J, Vammavaara E, Ek E, Laitinen J. Work ability index of young employees and their sickness absence during the following year. Scand J Work Environ Health 2006;32(1):75-84.

\begin{abstract}
Objectives The work ability index is widely used in occupational health care. The aim of this study was to determine the extent to which a low index among young employees is predictive of sickness absence during the following year.
\end{abstract}

Methods Altogether 3725 employees aged 31 years were followed from 1997 to 1999 as part of the northern Finland birth cohort 1966 study. The participants' self-administered questionnaires at baseline and a 1-year follow-up period with comprehensive sickness absence records was obtained from the Social Insurance Institution of Finland. The records contained only medically certified long-term sickness absences ( $>9$ days). A multivariable logistic regression was used to examine how the work ability index and covariates were associated with sickness absence during the following year. Odds ratios (OR) and 95\% confidence intervals (95\% CI) were calculated for the index and its covariates.

Results In a comparison with an excellent index score ( $>44$ points), a low score ( $<37$ points) was associated with sickness absence for both the men (OR 3.6, 95\% CI 1.6-7.9) and the women (OR 6.2, 95\% CI 2.9 to 13.5) after adjustment for occupation, control over work, social support at work, marital status, number of children at home, physical activity, body mass index, and alcohol consumption. Of the 518 participants with a low index, 90 had long-term sickness absence, giving a positive predictive value of $17.4 \%$ (95\% CI 14.1-20.6). The negative predictive value was $93.1 \%$ (95\% CI 92.3-94.0).

Conclusions For young employees, the work ability index is a practical tool for predicting long-term sickness absence, but it does not predict "no sickness absence".

Key terms classification of occupations; control over work; occupational health; longitudinal study; number of children; physical activity; prospective population-based study; questionnaire; sex factor; smoking.

The Work ability index developed by the Finnish Institute of Occupational Health (1) is a questionnaire-based method assessing perceived work ability. It was originally developed in a follow-up study of ageing employees (2). Despite the fact that the index was first developed to be used as a research method, it is nowadays widely used by occupational health professionals as a practical tool to measure subjective work ability.

Evidence of the acceptable internal validity of the index has been provided that shows a satisfactory relationship between the subjective results of the index and the results of more-objective measurements $(3,4)$. Furthermore, the satisfactory test-retest reliability of the index has been described (5). The index correlates with work stress factors and stress symptoms (6). Furthermore, it is a predictor of work disability among ageing employees $(7,8)$. The index is highly dependent on age $(9,10)$. The method has been well documented among mature-aged employees, but very little is known about the value of the index scores as an indicator of young adults' work ability. Reference values of the index for young employees have recently been introduced (11, 12). However, knowledge about the capability of the index to predict disability or sickness absence among young employees is still inadequate.

According to recent workforce surveys, the level of sickness absence has increased in western European countries (13), especially among women and older age

1 Finnish Institute of Occupational Health, Oulu, Finland

2 Oulu University Hospital, Outpatient Ward of Occupational Medicine, Oulu, Finland.

3 University of Oulu, Department of Public Health and General Practice, Oulu, Finland.

Correspondence to: Dr Veikko Kujala, Finnish Institute of Occupational Health, Aapistie 1, FI-90220 Oulu, Finland. [E-mail: veikko.kujala@ttl.fi] 
groups (14). Poor work conditions, such as low control over work (15-17) or heavy physical workload $(18,19)$, lead to a high sickness absence rate. Furthermore, sickness absence is also known to be related to factors other than health (20-22). Both research and regularly reported statistics show an association between socioeconomic status and sickness absence (23). In studies on sickness absence, it is often difficult to distinguish the factors that lead to a certain disease from the factors that lead to sickness absence. Sickness absence is a problem not only for the person suffering from the disease, but also for the immediate peer group, workplace, and the surrounding society.

This study evaluates the hypothesis that a decrease in the work ability index among young workers is associated with a higher risk of sickness absence in a 1-year follow-up and that the association is independent of educational level and control over one's work.

\section{Study population and methods}

In 1997, when the participants were in their early 30s, 3725 people were selected from the northern Finland birth cohort 1966 (NFBC-66) for a follow-up study. This follow-up study was carried out with a specific interest in evaluating long-term sickness absence. At baseline, participants were categorized into four work ability classes according to the work ability index. Individual sickness absence periods were then followed for 1 year using the only national register data, starting on the day after the participant had filled out the study questionnaire.

\section{Study population}

The participants were members of a population-based cohort, the NFBC-66 (24, 25). In 1997-1998, at the age of 31 years, altogether 11637 persons born in 1966 were alive, and questionnaires were mailed to all of those with known addresses (response rate 76\%, N=8767). In addition, 8463 persons who were still living in northern

Table 1. Seven items covered by the work ability index and the range of scores per item.

\begin{tabular}{lc}
\hline Item & $\begin{array}{c}\text { Range } \\
\text { of scores } \\
\text { (points) }\end{array}$ \\
\hline Current work ability compared with lifetime best & $0-10$ \\
Work ability in relation to the demands of the job & $2-10$ \\
Number of current diseases diagnosed by a physician & $1-7$ \\
Estimated work impairment due to diseases & $1-6$ \\
Sick leave during the past 12 months & $1-5$ \\
Personal prognosis of work ability 2 years from now & 1,4 or 7 \\
Mental resources & $1-4$ \\
\hline Total score & $7-49$ \\
\hline
\end{tabular}

Finland or had moved to the Helsinki capital area were also invited to the clinical examination, where an additional self-administered questionnaire was filled out (71\%, N=6033). The analyses were based on data for people who agreed to participate in the study and were employed at the age of 31 years. The responses of the unemployed $(\mathrm{N}=1390)$, those of people with missing data on current employment activity $(\mathrm{N}=325)$, and those of people who gave no written consent $(\mathrm{N}=43)$ for the use of data for research purposes were excluded from the analyses. Furthermore, 550 questionnaires were excluded due to missing data on items of the work ability index. Finally, 2021 men and 1704 women were included in the analyses. All of the procedures used in this study complied with the Declaration of Helsinki and were approved by the ethics committees of the Finnish Institute of Occupational Health and the University of Oulu.

\section{Assessment of sickness absence}

The main outcome variable was sickness absence. The data on medically certified sickness absences longer than 9 days were collected from the records kept by the Social Insurance Institution of Finland (SII) during 1997-1999. Individual sickness absences were followed for 1 year, and the participants with one or more sickness absences were counted. The sickness absence records contained no diagnostic information.

\section{Measure of the work ability index}

The work ability index of the young adults used in this study consisted of seven items, similar to the work ability index described previously $(1,7)$, as presented in Table 1. The participants completed a self-administered questionnaire during a clinical examination. The questionnaire used for the young adults in the present study listed a smaller number of illnesses than the original index. The following diseases were not included: myocardial infarction, pulmonary tuberculosis, hepatic or pancreatic disease, gastric or duodenal irritation, colonic irritation (colitis), and benign tumor. However, the following question was included: "Have you ever had other disease or injury diagnosed or treated by a physician?" For each item of the index, a single item score was obtained. The final score was a sum of all of the single-item scores, ranging from 7 to 49 points. The higher the score on the index, the better the work ability.

The participants' scores were classified into the following four categories: low, moderate, good, and excellent work ability. In categorizing, we used three cutoff points (the 15 th percentile, the median, and the 85 th percentile), based on the current distribution of the scores for young employees (12). By gender, this 
procedure resulted in $12 \%$ of the men and $16 \%$ of the women classified into the low work ability category (index scores 15-36), 27\% and 33\% into the moderate category (index scores $37-40$ ), $41 \%$ and $38 \%$ into the good category (index scores 41-44), and 20\% and 13\% into the excellent category (index scores 45-49).

\section{Definition of other explanatory and background variables}

The participants responded to questionnaires inquiring about marital status, number of children at home, occupation, and information about current employment activity, work characteristics, health behavior, and social support. Table 2 shows the characteristics of the participants by occupation, control over work, social support at work, marital status, number of children at home, physical activity, body mass index (BMI), smoking, and alcohol consumption.

The classification of occupations into nine major groups was based on the International Standard Classification of Occupations (ISCO-88) of the International Labour Organization with the exception that armed forces were merged with elementary occupations. In the categorization, the occupational groups were based on the nature of duties and tasks, related requirements, and the skill acquired either at work or through education. Major groups 2 to 9 were formed on the basis of skill level. In group 2, education takes 3 to 4 years or longer and leads to the lowest university degree, basic qualification, or the like. Occupations requiring a higher university degree belonged to group 2 . In group 3 , education lasts 4 years and leads to a qualification that is not equivalent to the lowest university degree. All jobs in major groups 4 to 8 belong to the same skill level with education lasting about 3 years.

Control over work was measured using internally consistent sets of 16 questions including skill discretion (6 items, eg, I have an opportunity to develop my own special abilities), vocational proficiency (1 item), authority to make decisions (6 items, eg, can you decide on how you do your work), and opportunities to participate in decision making ( 3 items, eg, I have a lot to say in matters concerning my workplace). Psychological demands of the work were addressed in nine items covering qualitative aspects, such as demands for attentiveness or precise discrimination. In order to keep the constructs of job demands and control over work separate, we included two items, one of skill discretion ("My job requires that I learn new things") and the other of decision making ("My job requires that I make decisions") in the demands of work instead of the control over work. These issues can be perceived by the respondents as more like demands, especially by young workers at the beginning of their careers $(26,27)$.
The variables describing work control and work demands were used as sum variables to create the four work control-demand categories described by Karasek (28). High and low control over work and job demands were defined by median cut-off points on the two summed scales. The categories were classified as "high strain" (high demands and low job control), "active" (high demands and high control), "passive" (low demands and low control), and "low strain" (low demands and high control). The amount of social support in the workplace was measured by means of a structured 5-point scale asking to what extent (a lot...not at all or not wanted) the participant received emotional support (listening or advice) or practical support (help with a worktask) from coworkers and supervisors (4 items). The sum of the four items was calculated and recorded as (i) little or no support, (ii) some support, and (iii) a lot of support.

The participants were classified into four groups by leisure-time physical activity according to their frequency, intensity, and duration of physical activity (29). Body weight and height were measured in a clinical examination, and the BMI $\left(\mathrm{kg} / \mathrm{m}^{2}\right)$ was calculated and classified into four groups (30), as shown in table 2. Furthermore, the questionnaire included questions on the frequency of the consumption of beer, wine, and spirits during the last year, and the usual amount of each per drinking occasion. The amount of alcohol consumed per day was calculated using the following alcohol contents (volume \%): beer 4.8, light wine 5.0, table wine 14.5, and spirits 37.0. This method was validated against 7 day food records (31).

\section{Statistical analysis}

Cross-tabulation was performed for sickness absence and each explanatory and background variable. The statistical significance of the comparisons was tested with Pearson's chi-square test. Univariate and multivariate binary logistic regression analyses were used to explore the association between the work ability index and sickness absence as the outcome variable. We used three multivariate logistic regression models with an enter procedure to study the association between the work ability index and sickness absence controlled for workrelated variables (occupational group, control over work, and social support at work, models I, II, III) + variables related to the family (marital status and number of children at home, models II and III) + BMI and health habits (physical activity, smoking, and alcohol consumption, model III). Odds ratios (OR) and their 95\% confidence intervals (95\% CI) are reported. A measure for explained variance was performed by Nagelkerke $\mathrm{R}^{2}$-value. All of the analyses were performed separately for the men and women using SPSS v. 12.0 software (SPSS, Chicago, IL, USA). 
Table 2. Characteristics of the participants of the northern Finland birth cohort (NFBC-66) study who were selected for sickness absence follow-up and the proportion of employees with sick leave of $>9$ days by gender in a 12-month period according to the characterizing variables. P-values of the chi-square test are presented for the characteristic to evaluate the association between the variable and sickness absence.

\begin{tabular}{|c|c|c|c|c|c|c|c|c|c|c|}
\hline \multirow[t]{3}{*}{ Characteristics } & \multicolumn{4}{|c|}{ Selected for follow-up } & \multicolumn{6}{|c|}{$\begin{array}{l}\text { Proportion of employees with sick leave } \\
\text { of }>9 \text { days in the 12-month period }\end{array}$} \\
\hline & \multicolumn{2}{|c|}{ Men } & \multicolumn{2}{|c|}{ Women } & \multicolumn{3}{|c|}{ Men } & \multicolumn{3}{|c|}{ Women } \\
\hline & $\mathrm{N}$ & $\%$ & $\mathrm{~N}$ & $\%$ & N & $\%$ & P-value & $\mathrm{N}$ & $\%$ & P-value \\
\hline Work ability index & - & . & - & . & - & . & $<0.001$ & - & . & $<0.001$ \\
\hline Low & 242 & 12 & 276 & 16 & 29 & 12 & $\cdot$ & 61 & 22 & . \\
\hline Moderate & 542 & 27 & 559 & 33 & 38 & 7 & . & 55 & 10 & . \\
\hline Good & 833 & 41 & 646 & 38 & 47 & 6 & . & 56 & 9 & . \\
\hline Excellent & 404 & 20 & 223 & 13 & 13 & 3 & $\cdot$ & 11 & 5 & . \\
\hline Occupation & - & . & - & . & - & . & 0.006 & - & . & 0.004 \\
\hline Legislators, senior officials and managers & 226 & 11 & 78 & 5 & 5 & 2 & . & 6 & 8 & . \\
\hline Professionals & 385 & 19 & 525 & 31 & 15 & 4 & . & 49 & 9 & . \\
\hline Technicians and associate professionals & 275 & 14 & 353 & 21 & 16 & 6 & . & 33 & 9 & . \\
\hline Clerks & 37 & 2 & 205 & 12 & 5 & 14 & . & 17 & 8 & . \\
\hline Service workers and shop or market sales workers & 134 & 7 & 266 & 16 & 14 & 10 & . & 32 & 12 & . \\
\hline Skilled agricultural and fishery workers & 159 & 8 & 80 & 5 & 12 & 8 & . & 16 & 20 & . \\
\hline Craft and related trades workers & 378 & 19 & 32 & 2 & 29 & 8 & . & 6 & 19 & . \\
\hline Plant and machine operators and assemblers & 280 & 14 & 63 & 4 & 17 & 6 & . & 14 & 22 & . \\
\hline Elementary occupations & 136 & 7 & 88 & 5 & 13 & 10 & $\cdot$ & 8 & 9 & $\cdot$ \\
\hline Control over work & - & . & - & - & - & $\cdot$ & $<0.001$ & - & . & 0.288 \\
\hline Active work & 641 & 33 & 496 & 31 & 28 & 4 & . & 46 & 9 & . \\
\hline High-strain work & 209 & 11 & 418 & 26 & 24 & 12 & . & 52 & 12 & . \\
\hline Low-strain work & 424 & 22 & 182 & 11 & 17 & 4 & . & 16 & 9 & . \\
\hline Passive work & 648 & 34 & 529 & 33 & 49 & 8 & $\cdot$ & 63 & 12 & $\cdot$ \\
\hline Social support at work & - & $\cdot$ & - & $\cdot$ & - & . & 0.364 & - & $\cdot$ & 0.052 \\
\hline Little & 434 & 22 & 290 & 17 & 33 & 8 & . & 40 & 14 & . \\
\hline Somewhat & 1161 & 59 & 945 & 57 & 66 & 6 & . & 86 & 9 & . \\
\hline A lot & 370 & 19 & 427 & 26 & 24 & 7 & . & 50 & 12 & . \\
\hline Marital status & - & . & - & . & - & . & 0.096 & - & . & 0.001 \\
\hline Married & 967 & 48 & 846 & 50 & 64 & 7 & . & 108 & 13 & . \\
\hline Cohabiting & 529 & 26 & 415 & 24 & 36 & 7 & . & 33 & 8 & . \\
\hline Single & 457 & 23 & 354 & 21 & 20 & 4 & . & 26 & 7 & . \\
\hline Divorced or widowed & 59 & 3 & 85 & 5 & 7 & 12 & $\cdot$ & 15 & 18 & . \\
\hline Number of children & - & . & - & . & - & . & $0.111^{\mathrm{a}}$ & - & . & $0.231^{\mathrm{a}}$ \\
\hline None & 854 & 44 & 610 & 37 & 45 & 5 & . & 61 & 10 & . \\
\hline One & 348 & 18 & 351 & 21 & 24 & 7 & . & 41 & 12 & . \\
\hline Two & 515 & 27 & 497 & 30 & 36 & 7 & . & 45 & 9 & . \\
\hline Three or more & 211 & 11 & 194 & 12 & 16 & 8 & $\cdot$ & 32 & 17 & $\cdot$ \\
\hline Physical activity & - & . & - & $\cdot$ & - & . & 0.239 & - & . & 0.081 \\
\hline Very active & 236 & 12 & 182 & 11 & 17 & 7 & . & 26 & 14 & . \\
\hline Active & 565 & 28 & 486 & 29 & 44 & 8 & . & 46 & 10 & . \\
\hline Moderately active & 580 & 29 & 591 & 35 & 34 & 6 & . & 73 & 12 & . \\
\hline Sedentary & 630 & 31 & 438 & 26 & 32 & 5 & . & 38 & 9 & . \\
\hline Body mass index $\left(\mathrm{kg} / \mathrm{m}^{2}\right)$ & - & . & - & . & - & . & $0.373^{\mathrm{a}}$ & - & . & $0.589^{a}$ \\
\hline Underweight $(<18.5)$ & 12 & 1 & 52 & 3 & 0 & 0 & . & 6 & 11 & . \\
\hline Normal weight (18.5-24.9) & 1011 & 50 & 1137 & 67 & 61 & 6 & . & 119 & 10 & . \\
\hline Overweight (25.0-29.9) & 827 & 41 & 374 & 22 & 52 & 6 & . & 40 & 11 & . \\
\hline Obese $(>29.9)$ & 168 & 8 & 130 & 8 & 14 & 8 & $\cdot$ & 17 & 13 & $\cdot$ \\
\hline Smoking & - & $\cdot$ & - & . & - & . & 0.434 & - & $\cdot$ & 0.269 \\
\hline Nonsmoker & 692 & 35 & 694 & 41 & 42 & 6 & . & 64 & 9 & . \\
\hline Ex-smoker & 425 & 21 & 406 & 24 & 25 & 6 & . & 50 & 12 & . \\
\hline Occasional smoker & 239 & 12 & 205 & 12 & 10 & 4 & . & 20 & 10 & . \\
\hline Current smoker & 645 & 32 & 390 & 23 & 46 & 7 & $\cdot$ & 48 & 12 & · \\
\hline Alcohol consumption & - & . & - & . & - & . & 0.500 & - & . & 0.553 \\
\hline Lowest 15\% & 296 & 15 & 250 & 15 & 17 & 6 & . & 26 & 10 & . \\
\hline Normal consumption & 1381 & 70 & 1165 & 70 & 84 & 6 & . & 130 & 11 & . \\
\hline Highest 15\% & 295 & 75 & 249 & 15 & 23 & 8 & . & 22 & 9 & . \\
\hline All & 2021 & 100 & 1704 & 100 & 127 & 6 & . & 183 & 11 & . \\
\hline
\end{tabular}

a Ordinal test. 
To obtain a positive predictive value for the work ability index, we included all of the participants with an index score categorized as low ( $<37$ points). Our reference standard was long-term sickness absence ( $>9$ days) during the 12-month follow-up. We calculated sensitivity and positive predictive values and estimated negative predictive values and specificity on the basis of the results.

\section{Results}

Altogether $8 \%$ of the employees (127 men and 183 women) had at least one sickness absence lasting $>9$ days during the year after the measurement of the work ability index. Of the employees classified as having excellent work ability, only $4 \%$ had at least one sickness absence. For the categories good, moderate, and low, the percentages were $7 \%, 8 \%$, and $17 \%$, respectively. There was an inverse gradient between the categorized score at 31 years of age and sickness absence among both genders (table 2). The lowest occurrence of sickness absence was found for those with the highest work ability score. Sickness absences were more common among the women than the men.

Table 2 and the crude odds ratios in table 3 show the association between sickness absence and the explanatory and background variables. For both genders,

Table 3. Multivariate logistic regression model of characteristics associated with sickness. (Adj = adjusted)

\begin{tabular}{|c|c|c|c|c|c|c|c|c|}
\hline & Crude OR & 2 $95 \% \mathrm{Cl}$ & Adj OR ${ }^{a}$ & $95 \% \mathrm{Cl}$ & Adj OR ${ }^{b}$ & $95 \% \mathrm{Cl}$ & Adj OR ${ }^{c}$ & $95 \% \mathrm{Cl}$ \\
\hline \multicolumn{9}{|l|}{ Men } \\
\hline \multicolumn{9}{|l|}{ Score for work ability } \\
\hline Low & 4.10 & $2.09-8.04$ & 3.42 & $1.62-7.23$ & 3.36 & $1.56-7.23$ & 3.63 & $1.64-8.05$ \\
\hline Moderate & 2.27 & $1.19-4.32$ & 1.97 & $0.97-4.01$ & 1.87 & $0.91-3.84$ & 1.96 & $0.95-4.07$ \\
\hline Good & 1.80 & $0.96-3.36$ & 1.94 & $0.98-3.81$ & 1.98 & $1.00-3.91$ & 1.85 & $0.92-3.71$ \\
\hline Excellent & 1.00 & .. & 1.00 &.. & 1.00 & .. & 1.00 & .. \\
\hline \multicolumn{9}{|l|}{ Occupation } \\
\hline Legislators, senior officials and managers & 1.00 & .. & 1.00 & .. & 1.00 & .. & 1.00 & .. \\
\hline Professionals & 1.79 & $0.64-5.00$ & 2.02 & $0.65-6.26$ & 2.01 & $0.65-6.27$ & 2.63 & $0.74-9.40$ \\
\hline Technicians and associate professionals & 2.73 & $0.99-7.57$ & 2.72 & $0.88-8.39$ & 2.94 & $0.95-9.09$ & 3.68 & $1.03-13.13$ \\
\hline Clerks & 6.91 & $1.89-25.19$ & 5.46 & $1.26-23.70$ & 6.14 & $1.40-26.99$ & 8.55 & $1.73-42.36$ \\
\hline Service workers and shop or market sales workers & s 5.16 & $1.81-14.66$ & 3.82 & $1.17-12.48$ & 3.74 & $1.14-12.29$ & 3.96 & $1.02-15.40$ \\
\hline Skilled agricultural and fishery workers & 3.61 & $1.25-10.46$ & 2.45 & $0.69-8.68$ & 2.29 & $0.62-8.48$ & 2.72 & $0.62-11.94$ \\
\hline Craft and related trades workers & 3.67 & $1.40-9.63$ & 3.45 & $1.16-10.31$ & 3.32 & $1.10-9.99$ & 4.47 & $1.29-15.53$ \\
\hline Plant and machine operators and assemblers & 2.86 & $1.04-7.87$ & 2.48 & $0.79-7.81$ & 2.40 & $0.75-7.68$ & 3.42 & $0.93-12.60$ \\
\hline Elementary occupations & 4.67 & $1.63-13.41$ & 4.83 & $1.49-15.69$ & 4.47 & $1.36-14.74$ & 5.11 & $1.33-19.64$ \\
\hline \multicolumn{9}{|l|}{ Control over work } \\
\hline Active work & 1.00 & .. & 1.00 & .. & 1.00 & .. & 1.00 & .. \\
\hline High-strain work & 2.84 & $1.61-5.02$ & 2.05 & $1.13-3.71$ & 2.18 & $1.19-3.98$ & 2.07 & $1.11-3.86$ \\
\hline Low-strain work & 0.91 & $0.49-1.69$ & 0.68 & $0.35-1.32$ & 0.74 & $0.38-1.42$ & 0.76 & $0.39-1.48$ \\
\hline Passive work & 1.79 & $1.11-2.89$ & 1.18 & $0.70-1.99$ & 1.23 & $0.72-2.11$ & 1.17 & $0.67-2.05$ \\
\hline \multicolumn{9}{|l|}{ Social support at work } \\
\hline Little & 1.19 & $0.69-2.05$ & 1.23 & $0.69-2.21$ & 1.12 & $0.62-2.03$ & 1.16 & $0.64-2.13$ \\
\hline Somehow & 0.87 & $0.54-1.41$ & 0.87 & $0.51-1.46$ & 0.77 & $0.45-1.31$ & 0.76 & $0.44-1.30$ \\
\hline A lot & 1.00 & .. & 1.00 & .. & 1.00 & .. & 1.00 & .. \\
\hline \multicolumn{9}{|l|}{ Marital status } \\
\hline Married & 1.00 & .. & . & . & 1.00 & .. & 1.00 & .. \\
\hline Cohabiting & 1.03 & $0.68-1.57$ & . & . & 1.17 & $0.71-1.94$ & 1.04 & $0.61-1.78$ \\
\hline Single & 0.65 & $0.39-1.08$ & . & . & 0.63 & $0.30-1.31$ & 0.61 & $0.29-1.29$ \\
\hline Divorced or widowed & 1.90 & $0.83-4.35$ & . & . & 1.51 & $0.53-4.27$ & 1.43 & $0.49-4.20$ \\
\hline \multicolumn{9}{|l|}{ Number of children } \\
\hline None & 1.00 & .. & . & . & 1.00 & .. & 1.00 & .. \\
\hline One & 1.33 & $0.80-2.22$ & . & . & 1.19 & $0.65-2.19$ & 1.24 & $0.65-2.36$ \\
\hline Two & 1.35 & $0.86-2.13$ & . & . & 1.11 & $0.61-2.01$ & 1.15 & $0.62-2.15$ \\
\hline More than two & 1.48 & $0.82-2.67$ & . & . & 1.25 & $0.59-2.66$ & 1.47 & $0.67-3.26$ \\
\hline \multicolumn{9}{|l|}{ Physical activity } \\
\hline Very active group & 0.92 & $0.51-1.64$ & . & . & . & . & 0.90 & $0.47-1.73$ \\
\hline Active group & 1.00 & .. & . & . & . & . & 1.00 & .. \\
\hline Moderately active group & 0.74 & $0.46-1.17$ & . & . & . & . & 0.60 & $0.35-1.03$ \\
\hline Inactive group & 0.63 & $0.40-1.01$ & . & . & . & . & 0.52 & $0.30-0.92$ \\
\hline \multicolumn{9}{|l|}{ Bocy mass index } \\
\hline Underweight $(<18.5)$ & 0.00 & .. & . & . & . & . & 0.00 & .. \\
\hline Normal weight (18.5-24.9) & 1.00 & .. & . & . & . & . & 1.00 & .. \\
\hline Overweight (25.0-29.9) & 1.04 & $0.71-1.53$ & . & . & . & . & 0.84 & $0.54-1.30$ \\
\hline Obese $(>29.9)$ & 1.43 & $0.78-2.61$ & . & $\cdot$ & . & . & 1.08 & $\begin{array}{l}0.53-2.20 \\
\quad(\text { continued) }\end{array}$ \\
\hline
\end{tabular}


Table 3. Continued.

\begin{tabular}{|c|c|c|c|c|c|c|c|c|}
\hline & Crude OR & $95 \% \mathrm{Cl}$ & Adj OR ${ }^{a}$ & $95 \% \mathrm{Cl}$ & Adj $\mathrm{OR}^{\mathrm{b}}$ & $95 \% \mathrm{Cl}$ & Adj OR ${ }^{c}$ & $95 \% \mathrm{Cl}$ \\
\hline \multicolumn{9}{|l|}{ Smoking } \\
\hline Nonsmoker & 1.00 & .. & . & . & . & . & 1.00 & .. \\
\hline Ex-smoker & 0.97 & $0.58-1.61$ & . & . & . & . & 0.77 & $0.44-1.36$ \\
\hline Occasional smoker & 0.68 & $0.33-1.37$ & . & . & . & . & 0.53 & $0.24-1.20$ \\
\hline Current smoker & 1.19 & $0.77-1.83$ & . & . & . & . & 0.91 & $0.54-1.55$ \\
\hline \multicolumn{9}{|l|}{ Alcohol consumption } \\
\hline Lowest $15 \%$ & 0.94 & $0.55-1.61$ & . & . & . & . & 0.87 & $0.46-1.67$ \\
\hline Normal consumption & 1.00 & .. & . & . & . & . & 1.00 & .. \\
\hline Highest 15\% & 1.31 & $0.81-2.11$ & $\cdot$ & $\cdot$ & $\cdot$ & $\cdot$ & 1.33 & $0.76-2.34$ \\
\hline \multicolumn{9}{|l|}{ Women } \\
\hline \multicolumn{9}{|l|}{ Score for work ability } \\
\hline Low & 5.47 & $2.80-10.68$ & 6.24 & $2.97-13.12$ & 5.97 & $2.82-12.66$ & 6.10 & $2.82-13.21$ \\
\hline Moderate & 2.10 & $1.08-4.10$ & 2.36 & $1.13-4.92$ & 2.26 & $1.08-4.74$ & 2.26 & $1.06-4.81$ \\
\hline Good & 1.83 & $0.94-3.56$ & 2.02 & $0.97-4.21$ & 1.87 & $0.89-3.92$ & 1.89 & $0.90-3.99$ \\
\hline Excellent & 1.00 &.. & 1.00 & .. & 1.00 & .. & 1.00 & .. \\
\hline \multicolumn{9}{|l|}{ Occupation } \\
\hline Legislators, senior officials and managers & 1.00 & .. & 1.00 & .. & 1.00 & .. & 1.00 & .. \\
\hline Professionals & 1.24 & $0.51-2.99$ & 1.24 & $0.46-3.30$ & 1.15 & $0.43-3.09$ & 1.16 & $0.43-3.14$ \\
\hline Technicians and associate professionals & 1.24 & $0.50-3.06$ & 1.24 & $0.45-3.39$ & 1.12 & $0.40-3.10$ & 1.07 & $0.38-2.99$ \\
\hline Clerks & 1.09 & $0.41-2.86$ & 1.00 & $0.34-2.96$ & 1.02 & $0.34-3.02$ & 0.96 & $0.32-2.90$ \\
\hline Service workers and shop or market sales workers & S 1.64 & $0.66-4.08$ & 1.46 & $0.53-4.04$ & 1.54 & $0.55-4.33$ & 1.64 & $0.58-4.68$ \\
\hline Skilled agricultural and fishery workers & 3.00 & $1.11-8.13$ & 1.44 & $0.45-4.60$ & 1.25 & $0.38-4.10$ & 1.33 & $0.40-4.42$ \\
\hline Craft and related trades workers & 2.77 & $0.82-9.35$ & 2.61 & $0.69-9.89$ & 2.59 & $0.66-10.11$ & 2.90 & $0.73-11.45$ \\
\hline Plant and machine operators and assemblers & 3.43 & $1.23-9.54$ & 2.91 & $0.93-9.17$ & 2.70 & $0.84-8.70$ & 3.06 & $0.94-10.01$ \\
\hline Elementary occupations & 1.20 & $0.40-3.62$ & 0.93 & $0.28-3.13$ & 0.98 & $0.29-3.35$ & 0.90 & $0.26-3.15$ \\
\hline \multicolumn{9}{|l|}{ Control over work } \\
\hline Active work & 1.00 & .. & 1.00 & .. & 1.00 & .. & 1.00 & .. \\
\hline High-strain work & 1.39 & $0.91-2.12$ & 1.32 & $0.84-2.08$ & 1.33 & $0.84-2.11$ & 1.39 & $0.86-2.23$ \\
\hline Low-strain work & 0.94 & $0.52-1.71$ & 0.87 & $0.46-1.65$ & 0.82 & $0.42-1.58$ & 0.90 & $0.46-1.76$ \\
\hline Passive work & 1.32 & $0.89-1.98$ & 1.14 & $0.72-1.81$ & 1.15 & $0.72-1.84$ & 1.13 & $0.70-1.84$ \\
\hline \multicolumn{9}{|l|}{ Social support at work } \\
\hline Little & 1.21 & $0.77-1.88$ & 1.10 & $0.68-1.78$ & 1.03 & $0.62-1.69$ & 1.01 & $0.61-1.69$ \\
\hline Somehow & 0.76 & $0.52-1.09$ & 0.68 & $0.46-1.01$ & 0.67 & $0.44-1.00$ & 0.62 & $0.41-0.95$ \\
\hline A lot & 1.00 & .. & 1.00 &.. & 1.00 & .. & 1.00 & .. \\
\hline \multicolumn{9}{|l|}{ Marital status } \\
\hline Married & 1.00 &.. & . & . & 1.00 & .. & 1.00 &.. \\
\hline Cohabiting & 0.59 & $0.39-0.89$ & . & . & 0.45 & $0.28-0.73$ & 0.46 & $0.28-0.76$ \\
\hline Single & 0.54 & $0.35-0.85$ & . & . & 0.39 & $0.22-0.68$ & 0.42 & $0.24-0.76$ \\
\hline Divorced or widowed & 1.46 & $0.81-2.65$ & . & . & 1.01 & $0.52-1.95$ & 1.08 & $0.55-2.16$ \\
\hline \multicolumn{9}{|l|}{ Number of children } \\
\hline None & 1.00 & .. & . & . & 1.00 & .. & 1.00 & .. \\
\hline One & 1.19 & $0.78-1.81$ & . & . & 0.76 & $0.47-1.23$ & 0.74 & $0.45-1.22$ \\
\hline Two & 0.90 & $0.60-1.34$ & . & . & 0.50 & $0.31-0.83$ & 0.50 & $0.30-0.85$ \\
\hline More than two & 1.78 & $1.12-2.82$ & . & . & 0.78 & $0.42-1.42$ & 0.83 & $0.44-1.56$ \\
\hline \multicolumn{9}{|l|}{ Physical activity } \\
\hline Very active group & 1.59 & $0.95-2.67$ & . & . & . & . & 1.79 & $1.00-3.19$ \\
\hline Active group & 1.00 & .. & . & . & . & . & 1.00 & .. \\
\hline Moderately active group & 1.35 & $0.91-1.99$ & . & . & . & . & 1.28 & $0.83-1.98$ \\
\hline Inactive group & 0.91 & $0.58-1.43$ & . & . & . & . & 0.64 & $0.38-1.08$ \\
\hline \multicolumn{9}{|l|}{ Bocy mass index } \\
\hline Underweight $(<18.5)$ & 1.07 & $0.45-2.56$ & . & . & . & . & 0.75 & $0.25-2.30$ \\
\hline Normal weight (18.5-24.9) & 1.00 & .. & . & . & . & . & 1.00 & .. \\
\hline Overweight (25.0-29.9) & 1.03 & $0.70-1.50$ & . & . & . & . & 0.90 & $0.59-1.37$ \\
\hline Obese $(>29.9)$ & 1.29 & $0.75-2.23$ & . & . & . & . & 0.99 & $0.51-1.89$ \\
\hline \multicolumn{9}{|l|}{ Smoking } \\
\hline Nonsmoker & 1.00 & .. & . & . & . & . & 1.00 & .. \\
\hline Ex-smoker & 1.38 & $0.93-2.05$ & . & . & . & . & 1.42 & $0.91-2.21$ \\
\hline Occasional smoker & 1.06 & $0.63-1.81$ & . & . & . & . & 1.13 & $0.63-2.04$ \\
\hline Current smoker & 1.38 & $0.93-2.05$ & . & . & . & . & 1.10 & $0.68-1.76$ \\
\hline \multicolumn{9}{|l|}{ Alcohol consumption } \\
\hline Lowest $15 \%$ & 0.92 & $0.59-1.44$ & . & . & . & . & 0.94 & $0.56-1.57$ \\
\hline Normal consumption & 1.00 & .. & . & . & . & . & 1.00 & .. \\
\hline Highest $15 \%$ & 0.77 & $0.48-1.24$ & . & . & . & . & 0.91 & $0.53-1.55$ \\
\hline
\end{tabular}

a Model with work-related characteristics $(\mathrm{N}=1580)$

${ }^{b}$ Model with work-related and family structure characteristics $(N=1530)$

c Model with work-related, family structure, lifestyle, living condition, and behavior characteristics ( $N=1482)$ 
occupational group was associated with sickness absence. Furthermore, divorced or widowed men and women had sickness absence more often than employees with another marital status. Among the women, the number of children at home ( 1 child or $\geq 3$ children) and social support at work were associated with sickness absence. Among the men, both work with high strain and passive work were associated with sickness absence. For the women, this association was weaker than for the men, and the Pearson chi-square test showed no statistical significance. There were no significant associations between physical activity, classified BMI, alcohol consumption, or smoking and sickness absence.

Table 3 shows the results of the multiple regression models predicting the long-term sickness absence among employees in their early 30s. Both the men and women who had low work ability showed increased odds ratios for sickness absence (figure 1). When occupation, control over work, and the characteristics of the family structure were included in the multivariate model (models I and II, table 3), the association between the work ability index and prolonged sickness absence was low among the men but somewhat stronger among the women. Further adjustments for physical activity, BMI, smoking, and alcohol consumption (model III) had slight effects on the odds ratios between the work ability index and sickness absence. All of the multivariate models presented in table 3 showed that the participants with low work ability at 31 years of age showed an increased risk of sickness absence in the following year.

Because sick leave during the past year (12 months) may predict later long-term sickness absence, we reevaluated our hypothesis using a modified work ability index without item 5 (sick leave during the past 12 months). The results showed that low work ability was associated with sickness absence among both the men (OR 2.6, 95\% CI 1.5-5.0) and the women (OR 4.3, 95\% CI 2.5-7.5), suggesting that the association between the work ability index and long-term sick leave during the following year is not fully explained by the number of sick leave days during the past 12 months that is selfreported in the questionnaire on work ability. Furthermore, when each item of the work ability index was evaluated by crude odds ratios, the goodness of fit $\left(\right.$ Nagelkerke $\mathrm{R}^{2}$ ) was between $6.3 \%$ (item 5 ) and $0.2 \%$ (item 7 , mental resources) for the observed effect on sickness absence. The $\mathrm{R}^{2}$ by item 1 , item 2 , item 3 , item 4 , and item 6 was $1.2 \%, 1.2 \%, 2.4 \%, 3.3 \%$, and $0.9 \%$, respectively.

Of the 518 participants with low work ability, 90 had long-term sickness absence, for a positive predictive value of $17.4 \%$ (95\% CI 14.1-20.6). The negative predictive value was $93.1 \%$ (95\% CI 92.3-94.0), specificity $87.5 \%$ (95\% CI $86.4-88.6$ ), and sensitivity $29.0 \%$ (95\% CI 24.0-34.1). The likelihood ratio was 2.3 (95\%
CI 1.9-2.8) for the occurrence of sickness absence and 0.8 (95\% CI 0.7-0.9) for no sickness absence.

\section{Discussion}

The results of this study provide a unique contribution to the literature on the work ability index, suggesting that measuring the work ability of young employees has a predictive value on long-term sickness absence. To our knowledge, this is the first report examining this relation in a comparatively young adult population. No previously published study has linked the work ability index and all-cause sickness absence. Young employees should be informed and encouraged to seek further occupational health care services if their work ability index is lower than 37 points. However, a negative likelihood ratio of 0.8 indicates that a score of 37 or more is not predictive of no sickness absence. Hence preventive measures aiming to reduce sickness absence should be offered to all employees.

The lower 15th percentile of the work ability index for young employees was about 10 points higher (37 versus 27 points) than for 44 - to 58 -year-old municipal employees (7). If the same cut-off point ( 27 points) is used between the poor and moderate work ability categories for young employees, there is a risk that the work ability of some persons with disability may be overestimated (12). In a study of home care workers in the city of Helsinki, the work ability index of a younger age group (19-34 years) was similar to that in this study. Among home care workers, only $1 \%$ of the young respondents were classified into the poor category (10).

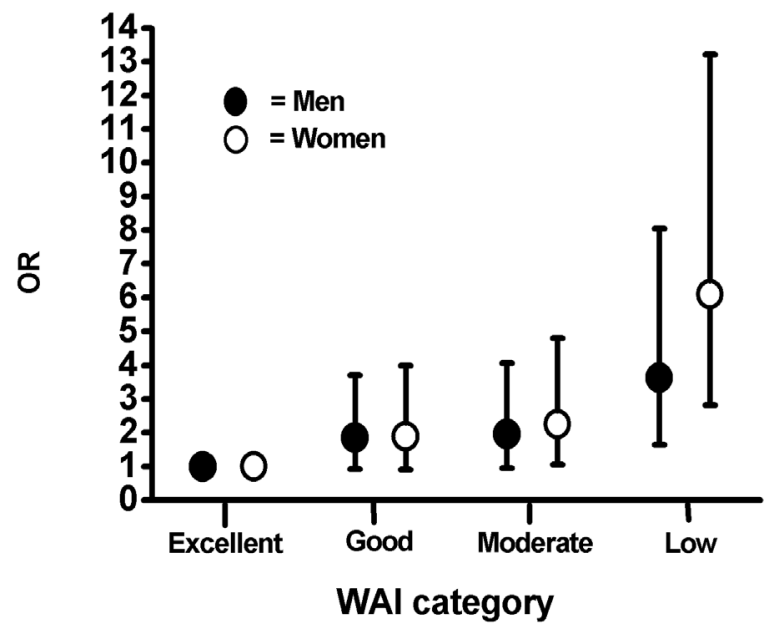

Figure 1. Odds ratios (OR) and 95\% confidence intervals for the men and women with sick leave of $>9$ days in a 12-month period according to their earlier work ability index (WAI) with adjustment for workrelated, family-structure, lifestyle, living condition, and behavior characteristics. 
The specific strengths of this study were the large sample size covering occupations in all kinds of enterprises and organizations, a study population with no significant age differences, determination of multiple indicators on health behavior and psychosocial factors at work, and the use of a reliable absence register. In Finland, employees receive full salary during sickness absences. At the same time, all employers receive statutory compensation for loss of salary due to sickness absence from the Social Insurance Institution of Finland. To receive the compensation, employers are obligated to show exact medical certifications in case of sickness absence, to keep precise records of sickness absences, and to send the medical certifications attached to the demand for compensation to the insurance institute. The Social Insurance Institution of Finland is the only national insurance institute in Finland that rules on the compensation for loss of salary due to temporary sickness absence. A limitation of this study was the lack of data on specific causes of sickness absence and shortterm sickness absences lasting fewer than 9 days.

The population was occupationally heterogeneous in our study. However, the study base enabled us to control for occupational groups. The association between occupation-based socioeconomic position and sickness absence is well known $(32,33)$. Among the men in their early 30 s, the two- to fivefold difference in the occurrence of prolonged sick leave between occupational group 1 and the other occupational groups was lower than described previously. In Great Britain (public administration, the Whitehall II study), North et al found (32) that people at the lowest socioeconomic level had 7.4 times as many long absences when compared with those at the highest level. Among the women, in accordance to previous findings, the difference between the major occupational groups was lower than among the men. In our study, the results showed a difference of up to 2.7-fold for long-term absences between occupational groups versus a 3.7-fold difference in the Whitehall II study (32).

As shown in figure 1, our findings suggest that a low work ability index is associated with gradually increasing sickness absences among young women and men across the occupational groups. The significant association between a low work ability index and sickness absence remained after control for occupation and other work features. Hence our results can be applied in all fields of work. Furthermore, the association is unlikely to result from obesity, smoking, alcohol consumption, or physical inactivity because the association remained even after control for such individual health behavior or known health risks. It is as important to recognize early signs of work disability among young workers as among ageing employees so that the requisite measures of occupational rehabilitation can be launched in time. The need to adjust the work to the worker's capability may require that the young employee improve his or her occupational skills or even find a new occupation. The results from this study emphasize that the work ability index provides an important source of information about health or the consequences of ill health among young workers as well.

The association between the psychosocial work environment and sickness absence is mostly based on Karasek's theory on the importance of demand and control. In the literature on sickness absence, there is evidence that low control of the work situation leads to higher sickness absence $(15,16)$. Our results support this hypothesis, especially for men in their early 30 s.

The finding of women's lower work ability is in accordance with prior research $(6,12,34)$. Furthermore, the finding of women's higher frequency of sickness absence agrees with the results of previous research (17, $32,35)$. These findings may reflect gender differences in morbidity, the perception of health, and behavior in response to illness $(20,36)$. The stronger association between the work ability index and sickness absence for women may indicate gender differences in attitudes towards work and long-term sickness absences.

The differences found between marital status categories are consistent with results from other studies, which show more sickness absences among divorced and separated men and women and widows and widowers than among married people $(37,38)$. Previous results with regard to children living at home are inconsistent. Our findings agree with the results of an earlier study showing that, in cases of absenteeism exceeding 7 days, there is a strong tendency for a higher occurrence of sickness absence among women with children under 10 years of age (39). However, no such association has been found in all studies (14).

Prior evidence shows that short-term sickness absences of 1 to 3 days are not associated with age, occupational status, or job insecurity in the same way that morbidity is $(20,40)$. Moreover, 1-day absences are more common on Mondays and Fridays than on other weekdays (22). According to the literature, short-term sickness absence has little to do with health. In our study, the outcome variable did not include short-term sickness absences. A self-administered questionnaire measuring perceived work ability seems to provide a tool for predicting long-term sickness absence.

Sickness absence rates are consequences of choices mediated by financial and other incentives. In addition to government-regulated national insurance, there is also the option of firm-workforce negotiation. As early as the 1950s, studies showed that workers who had access to compensation were more likely to be absent than others. Furthermore, higher compensation rates resulted in more absences. In the 1990s, it was also shown that 
higher rates led to longer absences (41). In countries in which there is no strong government insurance, these factors are of great importance. In our study, however, all of the participants were equally entitled to the benefit, and the differences in sickness absence cannot be explained by compensation rates. In Sweden, the introduction of a regulation shortening compensation coverage to 2 days or more resulted in an increase in the proportion of long ( $>15$ days) sickness absence periods (42).

Further research is needed on the generalizability of our findings to examine whether differences in sickness absence policies moderate the observed associations between work ability and sickness absence. Absence thresholds depend on the system of repayment (42), and further research is needed to determine whether our results are limited to the Finnish society. Furthermore, more research is needed to determine the predictive value of the work ability index for short-term sickness absences.

In conclusion, among young employees, the work ability index is a practical tool for predicting long-term sickness absence but is limited in predicting no sickness absence. We recommend that work ability be assessed in conjunction with periodic health check-ups or other types of screening procedures also among young employees. Professionals in occupational health care may provide a service for young workers with poor work ability (maximum score 36). Preventive measures aiming to reduce sickness absence should, however, be offered to all employees.

\section{References}

1. Tuomi K, Ilmarinen J, Jahkola A, Katajarinne L, Tulkki A. Työkykyindeksi [Work ability index]. Helsinki: Työterveyslaitos; 1997. Työterveyshuolto 19.

2. Tuomi K, Eskelinen L, Toikkanen J, Jarvinen E, Ilmarinen J, Klockars M. Work load and individual factors affecting work ability among aging municipal employees. Scand J Work Environ Health. 1991;17 Suppl 1:128-34.

3. Eskelinen L, Kohvakka A, Merisalo T, Hurri H, Wägar G. Relationship between the self-assessment and clinical assessment of health status and work ability. Scand J Work Environ Health. 1991;17 Suppl 1:40-7.

4. Nygård C-H, Eskelinen L, Suvanto S, Tuomi K, Ilmarinen J. Associations between functional capacity and work ability among elderly municipal employees. Scand J Work Environ Health. 1991;17 Suppl 1:122-7.

5. de Zwart BC, Frings-Dresen MH, van Duivenbooden JC. Testretest reliability of the Work Ability Index questionnaire. Occup Med (Lond). 2002;52:177-81.

6. Kloimüller I, Karazman H, Geissler I, Karazman-Morawetz I. The relation of age, work ability index and stress-inducing factors among bus drivers. Int J Ind Ergon. 2000;25:497-502.

7. Tuomi K, Ilmarinen J, Eskelinen L, Järvinen E, Toikkanen J,
Klockars M. Prevalence and incidence rates of diseases and work ability in different work categories of municipal occupations. Scand J Work Environ Health. 1991;17 Suppl 1:67-74.

8. Tuomi K, Ilmarinen J, Seitsamo J, Huuhtanen P, Martikainen R, Nygård C-H, et al. Summary of the Finnish research project (1981-1992) to promote the health and work ability of aging workers. Scand J Work Environ Health. 1997;23 Suppl 1:6671.

9. Chang YC, ChenSea MJ, Jang Y, Wang JD. A simple selfrating assessment method of residual work capability for occupational permanent disabilities. Am J Ind Med. 2000; 38:539-47.

10. Pohjonen T. Perceived work ability of home care workers in relation to individual and work-related factors in different age groups. Occup Med (Lond). 2001;51:209-17.

11. Kujala V, Väärälä M, Ek E, Tammelin T, Remes J, Laitinen J. Nuorten työssä käyvien työkykyindeksi [The work ability index of young employees in Finland]. Työ ja ihminen. 2002;16:308-22.

12. Kujala V, Remes J, Ek E, Tammelin T, Laitinen J. Classification of work ability index among young employees. Occup Med (Lond). 2005;55:399-401.

13. Alexanderson K, Norlund A. Aim, background, key concepts, regulations, and current statistics. Scand J Public Health Suppl. 2004:12-30.

14. Mastekaasa A. Parenthood, gender and sickness absence. Soc Sci Med. 2000;50:1827-42.

15. Bond FW, Bunce D. Job control mediates change in a work reorganization intervention for stress reduction. J Occup Health Psychol. 2001;6:290-302.

16. Ala-Mursula L, Vahtera J, Pentti J, Kivimäki M. Effect of employee worktime control on health: a prospective cohort study. Occup Environ Med. 2004;61:254-61.

17. Vahtera J, Kivimäki M, Pentti J, Linna A, Virtanen M, Virtanen $\mathrm{P}$, et al. Organisational downsizing, sickness absence, and mortality: 10-town prospective cohort study. BMJ. 2004;328:555.

18. Eshoj P, Jepsen JR, Nielsen CV. Long-term sickness absence-risk indicators among occupationally active residents of a Danish county. Occup Med (Lond). 2001;51:347-53.

19. Boedeker W. Associations between workload and diseases rarely occurring in sickness absence data. J Occup Environ Med. 2001;43:1081-8.

20. Marmot M, Feeney A, Shipley M, North F, Syme SL. Sickness absence as a measure of health status and functioning: from the UK Whitehall II study. J Epidemiol Community Health. 1995;49:124-30.

21. Briner RB. ABC of work related disorders: absence from work. BMJ. 1996;313:874-7.

22. Vahtera J, Kivimäki M, Pentti J. The role of extended weekends in sickness absenteeism. Occup Environ Med. 2001;58:818-22.

23. Allebeck P, Mastekaasa A. Risk factors for sick leave-general studies. Scand J Public Health Suppl. 2004;32:49-108.

24. Rantakallio P. Groups at risk in low birth weight infants and perinatal mortality. Acta Paediatr Scand. 1969;193:S1-71.

25. Rantakallio $P$. The longitudinal study of the northern Finland birth cohort of 1966. Paediatr Perinat Epidemiol. 1988;2:5988.

26. Kasl S. An epidemiological perspective on the role of job control in health, In: Sauter S, Hurrell J, Cooper C, editors. Job control and worker health. New York (NY): Wiley; 1989. p 161-89.

27. Nielsen M, Rugulies R, Christensen K, Smith-Hansen L, 
Bjorner J, Kristensen T. Impact of the psychosocial work environment on registered absence from work: a two-year longitudinal study using the IPAW cohort. Work Stress. 2004;18:323-35.

28. Karasek R. Job demands, job decision latitude and mental strain: Implications for job redesign. Adm Sci Q. 1979;24:285-308.

29. Tammelin T, Näyhä S, Hills AP, Järvelin M-R. Adolescent participation in sports and adult physical activity. Am J Prev Med. 2003;24:22-8.

30. World Health Organization (WHO). Obesity preventing and managing the global epidemic. Geneva: WHO; 1998.

31. Laitinen J, Pietiläinen K, Wadsworth M, Sovio U, Järvelin MR. Predictors of abdominal obesity among 31-y-old men and women born in Northern Finland in 1966. Eur J Clin Nutr. 2004;58:180-90.

32. North F, Syme SL, Feeney A, Head J, Shipley MJ, Marmot MG. Explaining socioeconomic differences in sickness absence: the Whitehall II Study. BMJ. 1993;306:361-6.

33. Fuhrer R, Shipley MJ, Chastang JF, Schmaus A, Niedhammer I, Stansfeld SA, et al. Socioeconomic position, health, and possible explanations: a tale of two cohorts. Am J Public Health. 2002;92:1290-4.

34. Tuomi K, Ilmarinen J, Martikainen R, Aalto L, Klockars M. Aging, work, life-style and work ability among Finnish municipal workers in 1981-1992. Scand J Work Environ Health. 1997;23 Suppl 1:58-65.

35. Elovainio M, Kivimäki M, Vahtera J. Organizational justice: evidence of a new psychosocial predictor of health. Am J Public Health. 2002;92:105-8.

36. Mustard CA, Kaufert P, Kozyrskyj A, Mayer T. Sex differences in the use of health care services. N Engl J Med. 1998;338:1678-83.

37. Leigh JP. Employee and job attributes as predictors of absenteeism in a national sample of workers: the importance of health and dangerous working conditions. Soc Sci Med. 1991;33:127-37.

38. Rael EG, Stansfeld SA, Shipley M, Head J, Feeney A, Marmot M. Sickness absence in the Whitehall II study, London: the role of social support and material problems. J Epidemiol Community Health. 1995;49:474-81.

39. Åkerlind I, Alexanderson K, Hensing G, Leijon M, Bjurulf P. Sex differences in sickness absence in relation to parental status. Scand J Soc Med. 1996;24:27-35.

40. Vahtera J, Kivimäki M, Koskenvuo M, Pentti J. Hostility and registered sickness absences: a prospective study of municipal employees. Psychol Med. 1997;27:693-701.

41. Barnby T, Ercolani M, Treble J. Sickness absence: an international comparison. Econ J. 2002;112:315-32.

42. Voss M, Floderus B, Diderichsen F. Changes in sickness absenteeism following the introduction of a qualifying day for sickness benefit-findings from Sweden Post. Scand J Public Health. 2001;29:166-74.

Received for publication: 19 April 2005 